\title{
CRITERIA OF LOW-NOISE THICK-FILM RESISTORS
}

\author{
L. K. J. VANDAMME \\ Eindhoven University of Technology, Department of Electrical Engineering, Eindhoven, Netherlands
}

(Received June 8, 1977)

\begin{abstract}
Our simple model of the noise of a thick-film resistor leads to two limiting cases. For thick-film resistors with a conduction dominated by the glass interface, the relative noise is proportional to the sheet resistance, $R_{\square}$. For thick film resistors, where the conduction is mainly dominated by the current constrictions in the contact areas between grains, the relative noise is proportional to $R_{\square}^{3}$. Both trends have been observed. Some criteria of low-noise thickfilm resistors are derived from the developed noise relations.

The major conclusion is that measurements of the noise index are a nondestructive way to check quantitatively the operations used to manufacture high-quality thick-film resistors. Inadequate materials or treatments, weak or brittle wire bonds and reliability are easily detected by $1 / \mathrm{f}$ noise measurements.
\end{abstract}

\section{INTRODUCTION}

A qualitative study of the noise index in thick-film resistors has been made ${ }^{1,2}$ and the effects of trimming and resistor geometry on the noise index have previously been investigated. ${ }^{3-5}$

Tiny contacts between grains in an aggregate structure cause local increase in the current density on a microscopic scale. The noise in the voltage across electrodes is proportional to the integral $\int_{\mathrm{v}}|J|^{4} \mathrm{dv}$, integrated over the volume between the electrodes through which a constant current is passed. Trim cuts lead to an increase in local current density and so to an increase in the noise of a thickfilm resistor. The noise at the conductor resistor interface has been attributed to micro cracks in this interface. Patchy contacts at a metal-semiconductor interface have been studied quantitatively by using contact resistance and noise measurements. ${ }^{6} 1 / \mathrm{f}$ noise measurements were recommended as a diagnostic tool for research on thick-films, ${ }^{1,7}$ for thinfilm metalization patterns ${ }^{8}$ and for quantitative characterization of contacts in general. ${ }^{6,9}$ Considering the distribution of metal oxide grains in the poor conducting matrix, most thick-film resistors are homogeneous. The aggregate structure of grains joined by narrow necks causes a meandering path for the current through grains and linking branches of glass. On a microscopic scale the current density is non-uniform. This fact leads to an increase in the noise voltage. The $1 / \mathrm{f}$ noise in such thick-film resistors will be discussed here in terms of arrays of contacts in parallel and in series. Apart from a geometric factor, the noise behaviour of a thick-film resistor is similar to that of a single contact. Hooge and Hoppenbrouwers ${ }^{10}$ have shown that contact $1 / \mathrm{f}$ noise is not a special type of noise but is physically the same as bulk $1 / \mathrm{f}$ noise. We have developed a model of the $1 / \mathrm{f}$ noise in the contact resistance between touching bodies affected by poor conducting layers ${ }^{1,12}$ and of patchy contacts consisting of an assembly of conducting spots $^{6,9}$ in parallel. Noise expressions have already been derived ${ }^{13}$ for a channel-like conducting branch.

\section{1/f NOISE MODEL FOR THICK-FILM RESISTORS}

For the sake of simplicity we carry out the calculations for a resistor thick-film as illustrated in Figure 1. The length of the sample between the terminals is $l$, the breath and the thickness are given by $b$ and $d$. The number of grain layers in the length, breath and thickness directions are $k_{l}, k_{b}$ and $k_{d}$. The relative size of grains or gaps between grains filled by the vitrious phase have not been drawn to scale. The following assumptions are made: (i) the major part of the conduction takes place through linking branches in the length direction, (ii) the conductor-resistor interface is an ideal contact, so the derived resistance and noise formulae refer to the resistor material between the conductors, (iii) the resistance $r$ and the average relative resistance fluctuations $\left\langle(\Delta r / r)^{2}>\right.$ for all linking branches have the same values, (iv) the fluctuations produced in all 


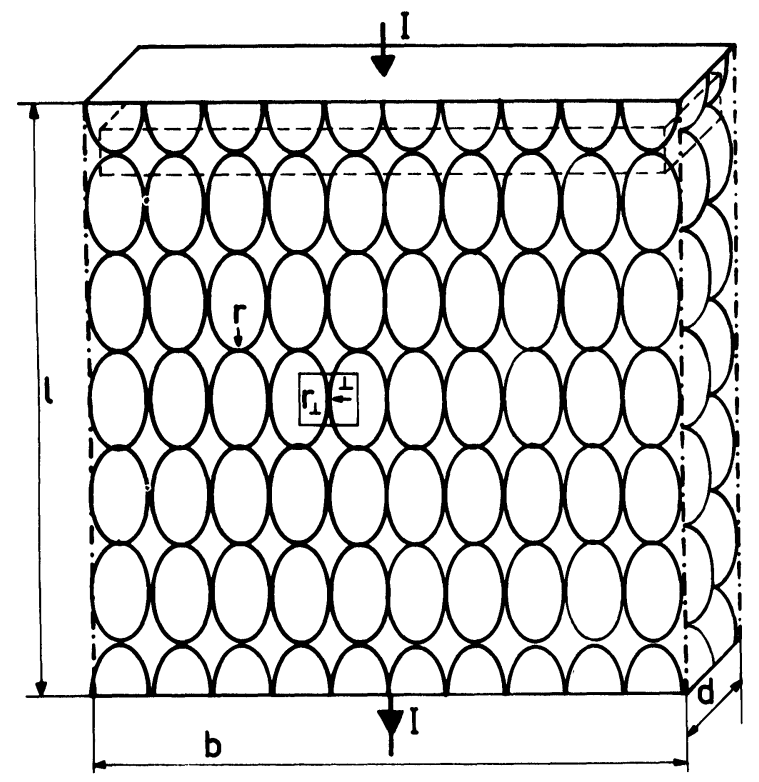

FIGURE 1 Simple model for a thick-film resistor.

linking branches are uncorrelated, (v) the resistance $r$ of the type represented in Figure 1 by the area labelled $\perp$ are assumed to carry no net current. Then the fluctuations $\left\langle\left(\Delta r_{\perp}\right)^{2}>\right.$ do not contribute to the resistance noise $<(\Delta \mathrm{R} / \mathrm{R})^{2}>$ between the conductors. ${ }^{14}$ The influence of side-paths carrying net current can be taken into account, by considering $r$ in parallel with $r_{\perp}$ and by adding the fluctuations of the conductance paths in parallel. In this way a new $r_{\text {eff }}$ and $\left\langle\left(\Delta r_{e f f}\right)^{2}\right\rangle$ are obtained. A discussion on the noise of unequal conduction paths in parallel has been given. ${ }^{15}$

The resistance $\mathrm{R}_{l}$ of a layer perpendicular to the length direction and consisting of $k_{b} \times k_{d}$ grain contacts becomes $\mathrm{R}_{l}=r / k_{b} k_{d}$ where $r$ is the resistance of one single conducting path. The total conductance $\mathrm{G}_{l}=1 / \mathrm{R}_{l}$ of such a layer equals the sum of all grain contact conductances $g=1 / r$ in parallel. The fluctuations $\Delta \mathrm{G}_{l}$ are obtained as the sum over the fluctuations $\Delta g_{i}$ of all grain contacts in parallel. The average of the squared fluctuating layer conductance $<\left(\Delta \mathrm{G}_{l}\right)^{2}>$ equals the average of the squared sum of all fluctuations $\Delta g_{i}$. Since the fluctuations are assumed to be uncorrelated the average values of the cross products $\left\langle\Delta g_{i} . \Delta g_{j}\right\rangle$ are zero. Since all $\left\langle\left(\Delta g_{i}\right)^{2}>\right.$ are assumed to have the same value $\left\langle\left(\Delta \mathrm{G}_{l}\right)^{2}\right\rangle=k_{b} k_{d}\left\langle(\Delta g)^{2}\right\rangle$. For small relative fluctuations it is easily seen that $\left\langle\left(\Delta \mathrm{G}_{l} / \mathrm{G}_{l}\right)^{2}\right\rangle=\left\langle\left(\Delta \mathrm{R}_{l} / \mathrm{R}_{l}\right)^{2}\right\rangle$ and $\left\langle(\Delta r / r)^{2}\right\rangle=$ $<(\Delta g / g)^{2}>$. So the relative noise for the layer resistance becomes $\left\langle\left(\Delta \mathrm{R}_{l} / \mathrm{R}_{l}\right)^{2}\right\rangle=\left\langle(\Delta r / r)^{2}\right\rangle \mid$ $k_{b} k_{d}$. The resistance $\mathrm{R}$ between the conductors consists of $k_{l}$ grain contact layers in series and becomes

$$
\mathrm{R}=\left(k_{l} / k_{b} k_{d}\right) r
$$

Following the same reasoning as above, leads to a relation for the noise $\left\langle(\Delta \mathrm{R})^{2}\right\rangle=k_{l}\left\langle\left(\Delta \mathrm{R}_{l}\right)^{2}\right\rangle$. The relative noise in the resistance $R$ becomes

$$
\left.<(\Delta \mathrm{R} / \mathrm{R})^{2}\right\rangle=\left\langle(\Delta r / r)^{2}>/ k_{l} k_{b} k_{d}\right.
$$

The relative noise $<(\Delta R / R)^{2}>$ equals the relative noise $\left\langle(\Delta r / r)^{2}\right\rangle$ of a single linking branch multiplied by a reducing factor $1 / k_{l} k_{b} k_{d}$.

Introducing the quantities $\gamma_{l}, \gamma_{b}$ and $\gamma_{d}$ each representing the number of linking branches per unit length in the length, breadth and thickness directions, the geometry factors in Eqs. (1) and (2) are written in the form of the resistor dimensions $l, b$ and $d$. Assuming a homogeneous distribution of the grains, the factors in Eqs. (1) and (2) become $\left(\gamma_{l} / \gamma_{b} \gamma_{d}\right)(l / b d)$ and $1 /\left(\gamma_{l} \gamma_{b} \gamma_{d} l b d\right)$ respectively. It is clear that the relative noise $<(\Delta R / R)^{2}>$ is inversely proportional to the volume l.b.d. If $l=b$, then Eq. (2) represents the relative noise of the sheet resistance $<\left(\Delta R_{\square}\right)-$ $\left.\mathrm{R}_{\square}\right)^{2}>$ which is (in contradistinction to the sheet resistance $R_{\square}$ itself) inversely proportional to the area $l^{2}$. The inverse proportionality between the relative noise (noise index) and the volume of the resistor is in agreement with experiments. ${ }^{4,16}$ A simple analysis makes it possible to calculate $\gamma_{l} / \gamma_{b}$ and $r / r_{\perp}$ from experimentally obtained anisotropy ratios in the sheet resistance and in the relative noise. This will be carried out for carbon sheet resistors in section 3.3. For isotropic resistors such as thick-film resistors, the number of contacts per unit length between grains is assumed to be $\gamma_{l}=\gamma_{b}=$ $\gamma_{d}=\gamma$, and if a square area is considered, then Eqs. (1) and (2) reduce to

$$
\begin{aligned}
& \mathrm{R}_{\square}=r / d \gamma \\
& \left.<\left(\Delta \mathrm{R}_{\square} / \mathrm{R}_{\square}\right)^{2}\right\rangle=\left\langle(\Delta r / r)^{2}>/ \gamma^{3} l^{2} d\right.
\end{aligned}
$$

Our first criterion of low-noise thick-film resistors is to use small grains, which can result in great values of $\gamma$. Using smaller grains and assuming that this leads to greater $\gamma$ values, the $R_{\square}$ values become smaller if $d$ and $r$ are kept constant. The noise $\left\langle\left(\Delta R_{\square} / R_{\square}\right)^{2}\right\rangle$ decreases proportionally to decreasing $R_{\square}{ }^{3}$. For the same $\gamma$ and $r$ but thicker samples, $\angle\left(\Delta R_{\square} / R_{\square}\right)^{2}>$ is proportional to $R_{\square}$. 
A second criterion is to use materials for which the "noise source" $\left\langle(\Delta r / r)^{2}\right\rangle$ is low. If we can choose between two resistive materials of equal resistivity, the one with lower carrier mobility and hence higher free carrier concentration will be favoured for getting low $1 / \mathrm{f}$ noise. This is a result of Hooge's empirical relation ${ }^{17}$ given by

$$
\left.<(\Delta \mathrm{R} / \mathrm{R})^{2}\right\rangle=\mathrm{C} \Delta \mathrm{f} / \mathrm{f} \text { with } \mathrm{C}=\alpha / \mathrm{N}
$$

where $\alpha$ is about $2 \times 10^{-3}, \mathrm{~N}$ the total number of free charge carriers and $\left\langle(\Delta R / R)^{2}\right\rangle$ is the average of the squared relative resistance fluctuation observed in a band $\Delta f$ centered at frequency $f$. The empirical relation in this form holds for homogeneous metal and semiconductor samples subjected to uniform fields. A variant of $\mathrm{C}=\alpha / \mathrm{N}$ in terms of the sample resistance $\mathrm{R}$ and its length $l$ is given by

$$
\mathrm{C}=\alpha \mathrm{q} \mu \mathrm{R} / l^{2}
$$

where $\mu$ is the mobility of the free charge carriers.

The relative $1 / \mathrm{f}$ noise intensity is denoted by $\mathrm{C}$ and this is the value of the relative power density spectrum at $1 \mathrm{~Hz}$. Now two situations will be considered. Simplified conceptual models are illustrated in Figures 2a and 2b. In Figure 2a the

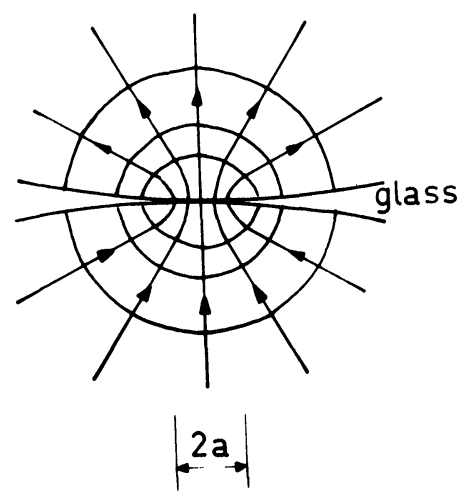

FIGURE 2a Constriction dominated contact between two grains.

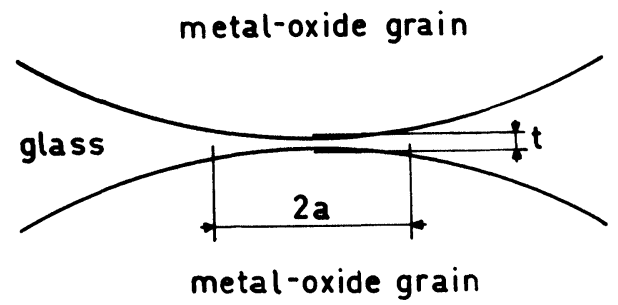

FIGURE 2b Interface dominated contact between two grains. qualitative shape of current flows and equipotential lines are represented for two touching grains.

We assume a circular contact spot with radius $a$ and homogeneous conductivity in the grains. The resistance, $r$, equals $\rho / \pi a$ and the relative fluctuations

$$
<(\Delta r / r)^{2}>=\alpha \pi^{2} \mathrm{r}^{3} \Delta \mathrm{f} / 20 \mathrm{n} \rho^{3} \mathrm{f}
$$

where $\rho$ is the resistivity of the metal oxide grains, and $n$ is the free charge carrier concentration. ${ }^{10,13}$ The substitution of $r$ and $\left\langle(\Delta r / r)^{2}\right\rangle$ in Eqs. (3) and (4) leads to the following results:

$$
\begin{aligned}
& \mathrm{R}_{\square}=\rho / \pi \mathrm{a} d \gamma \\
& \begin{aligned}
<\left(\Delta \mathrm{R}_{\square} / \mathrm{R}_{\square}\right)^{2}> & =\alpha \pi^{2} \mathrm{R}_{\square}^{3} d^{2} \Delta \mathrm{f} / 20 \mathrm{n} \rho^{3} l^{2} \mathrm{f} \\
& =\mathrm{C}_{u s} \Delta \mathrm{f} / \mathbb{R}^{2} \mathrm{f}
\end{aligned}
\end{aligned}
$$

where $C_{u s}$ is the relative $1 / f$ noise intensity for a square of unit area.

In Figure $2 b$ a contact affected by a uniform (nonpunched) interface is presented. The resistance $r$ now consists of constriction resistance equal to $\rho / \pi a$ and an interface resistance equal to $t \rho_{\mathrm{i}} / \pi a^{2}$, where $t$ is an effective thickness, which is about the distance between the nearest grain surface, and $a$ is an effective radius, which is greater than the radius of the circular contact spot $a$ if no interface layer is present. The resistivity of the interface layer is $\rho_{\mathrm{i}}$. The ratio $t \rho_{\mathrm{i}} / a \rho$ determines whether a contact and consequently a thick-film resistance is constriction dominated or interface dominated. The noise of such a model has been studied. ${ }^{11}$ For high values of the ratio $t \rho_{\mathrm{i}} / a \rho, r$ equals $\rho_{\mathrm{i}} t / \pi a^{2}$ and $<(\Delta r / r)^{2}>$ equals $\alpha q \mu_{\mathrm{i}} r \Delta \mathrm{f} / t^{2} \mathrm{f}$, where $\mathrm{q}$ is the absolute value of the charge of an electron and $\mu_{\mathrm{i}}$ is the mobility of free charge carriers in the interface. The substitution of $r$ and $\left\langle(\Delta r / r)^{2}\right\rangle$ in Eqs. (3) and (4) leads to the following results:

$$
\begin{aligned}
\mathrm{R}_{\square}=\rho_{\mathrm{i}} t / \pi a^{2} \gamma d & \\
<\left(\Delta \mathrm{R}_{\square} / \mathrm{R}_{\square}\right)^{2}> & =\alpha \mathrm{q} \mu_{\mathrm{i}} \mathrm{R}_{\square} \Delta \mathrm{f} / \gamma^{2} l^{2} t^{2} \mathrm{f} \\
& =\mathrm{C}_{u s} \Delta \mathrm{f} / l^{2} \mathrm{f}
\end{aligned}
$$

Here the relative $1 / f$ noise intensity for a square of unit area is proportional to $R_{\square}$.

\section{EXPERIMENTAL RESULTS}

\subsection{The Noise Measuring Set-up}

The block diagram of the electrical measuring set-up 
is given in Figure 3. A constant current $I$ is passed through the sample $R$. This current is derived from a fresh battery or cell in series with a quality resistor $R_{v}$. A low noise a.c. pre-amplifier is connected with the sample. The amplified noise is then passed through a set of nine band-pass filters with the input terminals in parallel. The fixed central frequencies $\mathrm{f}_{\mathrm{o}}$ range from $10 \mathrm{~Hz}$ to $100 \mathrm{kHz}$. The output signals can be selected to the input of an amplifier. Such an arrangement can be called a semi real-time spectrum analyser. The band-pass filters are $6^{\text {th }}$ order Butterworth filters ( 3 sections) some with $\Delta f / f_{o}$ equal to $10 \%$ and others with $\Delta \mathrm{f} / \mathrm{f}_{\mathrm{o}}$ equal to $100 \%$, where $\Delta \mathrm{f}$ is the $3 \mathrm{db}$ bandwidth of the filter. The filters with central frequencies of $316 \mathrm{~Hz}$ and higher are passive filters, the other ones with lower $f_{o}$ are active filters. The filtered and amplified noise is passed through a squaring module. The squared signal is then passed through a low-pass filter giving a running-time average of the squared and filtered noise. A digital voltmeter and a recorder are used for displaying the signal.

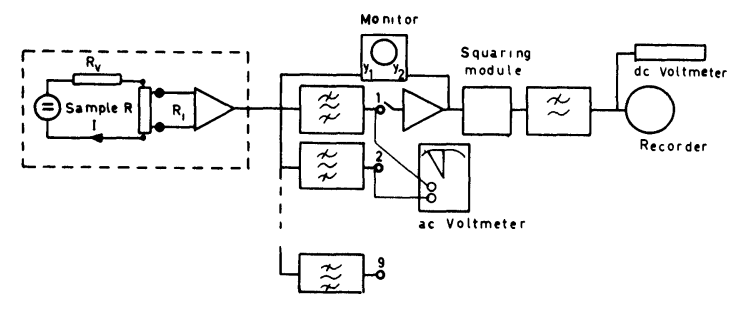

FIGURE 3 Diagram of the noise measuring set-up.

The dotted line in Figure 3 represents the shielding case. Grounding and shielding practice are treated by Morrison. ${ }^{18}$

The channel $\mathrm{y}_{1}$ of the oscilloscope is used as a noise monitor. In this way hum, burst noise, or low frequency oscillations and clipping of the preamplifier can easily be detected. The second channel $\mathrm{y}_{2}$ is used as monitor for the filtered and amplified noise.

The two-channel a.c. voltmeter is used as time saver for a quick $1 / \mathrm{f}$ spectrum check. Two band-pass filters with the same relative bandwidth $\Delta \mathrm{f} / \mathrm{f}_{\mathrm{o}}$ give the same r.m.s. voltage if a pure $1 / \mathrm{f}$ spectrum is passed through the filters with the input terminals in parallel. Electronic current sources were not used. A low resistance $R$ requires high currents and batteries were used in series with a load resistor $R_{v}$. For the other samples lower currents from dry cells were used. Noise characteristics of batteries and dry cells are given by Euler. ${ }^{19}$ By using wire-wound, metal-film or carbon-film resistors for $\mathrm{R}_{\mathrm{v}}$ a constant current is established. Solid carbon composition resistors were avoided because of their appreciable $1 / \mathrm{f}$ noise. By replacing the sample by a wire wound resistor of the same resistance, it can be checked whether the current is noiseless.

If the sample resistance is of the same order as the input resistance $R_{i}$ of the pre-amplifier then a constant voltage over the sample can be used instead of a constant current through the sample. Then $R_{v}$ and $R$ in Figure 3 are interchanged while now $50 R_{v}<R$. The fluctuating voltage across $R_{v}$ is measured. If the sample resistance is at least 50 times greater than the small resistor $R_{v}$ then the fluctuating voltage $\left\langle(\Delta \mathrm{I})^{2}>\mathrm{R}^{2}\right.$ equals $\mathrm{I}^{2}<(\Delta \mathrm{R})^{2}>$ and hence the relative voltage fluctuation measured across the small series resistance $\left\langle(\Delta \mathrm{V} / \mathrm{V})^{2}\right\rangle$ is equal to $\left\langle(\Delta \mathrm{R} / \mathrm{R})^{2}\right\rangle$.

For low resistance samples $(\mathrm{R}<300 \Omega)$ a preamplifier with an input resistance of $10 \mathrm{k} \Omega$ is used. Samples with a resistance greater than $300 \Omega$ are measured with a low noise Brookdeal amplifier (model 453). The former pre-amplifier has a $3 \mathrm{db}$ bandwidth ranging from $1 \mathrm{~Hz}$ to $100 \mathrm{kHz}$ and an equivalent input noise resistance $R_{e}$ of $30 \Omega$ above $1 \mathrm{kHz}$. The latter had a $3 \mathrm{db}$ bandwidth ranging from $1 \mathrm{~Hz}$ to $1 \mathrm{MHz}$ and an equivalent input resistance of $1 \mathrm{k} \Omega$ above $300 \mathrm{~Hz}$. The first stage of the preamplifier with $R_{e}$ equal to $30 \Omega$ is made up of eight selected input transistors in parallel (PNP BC214). Transistors in parallel improve the signal-to-noise ratio because the noise powers from each transistor will add, whereas the signal amplitudes will add.

The systematic deviations (instrument errors) in the measured spectra due to the finite bandwidth of the band-pass filter can easily be estimated. Let us assume that the smooth spectrum has the form

$$
\mathrm{S}_{\mathrm{v}}(\mathrm{f})=\mathrm{Cf}^{-h}
$$

where $h$ is independent of the frequency. This spectrum is then measured by a sharp band-pass filter with infinite attenuation outside the band-pass frequency. The central frequency, the low and high cut-off frequencies are $f_{o}, f_{L}=f_{o} / \sqrt{ } s$ and $f_{h}=f_{o} \sqrt{ } s$ respectively where $s>1$. Hence, we obtain $f_{o}^{2}=f_{L} f_{h}$ and $f_{h}=s f_{L}$ and $\left(f_{h}-f_{L}\right) / f_{o}=\Delta f / f_{o}=(s-1) / \sqrt{ } s$. For $s=2$ we have a so-called octave band-pass filter with a relative bandwidth $\Delta f / f_{o}$ of $70 \%$. The often used $1 / 3$ octave band-pass filter has $\Delta f / f_{o}=70.7 / 3=$ $23.6 \%$ and $s$ is about 1.265 . The measured values $\widetilde{\mathrm{S}}_{\mathrm{v}}$ of the spectrum given in Eq. (12) for $h=1$ is 
then

$$
\begin{aligned}
\widetilde{S}_{v}\left(f_{o}\right)=\frac{1}{f_{o}(\sqrt{s}-1 / \sqrt{ } s)} \int_{f_{o} / \sqrt{s}}^{f_{o} \sqrt{s}} \\
\frac{\operatorname{Cdf}}{f}=S_{v}\left(f_{o}\right) \frac{1 n s}{s-1 / \sqrt{ } s}
\end{aligned}
$$

From Eq. (13) we see that $1 / 3$ octave band-pass filters and filters with smaller relative bandwidth $\widetilde{S}_{V}\left(f_{o}\right)=S_{v}\left(f_{o}\right)$, even for $\Delta f / f_{o}=100 \%(s=2.618)$ $\mathrm{S}_{\mathrm{v}}\left(\mathrm{f}_{\mathrm{o}}\right)$ is only $4 \%$ smaller than $\mathrm{S}_{\mathrm{v}}\left(\mathrm{f}_{\mathrm{o}}\right)$.

\subsection{Experimental Results on Thick-film Resistors}

From the definition of the Index in an ideal decade pass-band $\mathrm{d}^{20}$ and from Eq. (13) it follows that

$$
\text { Index } \mathrm{dB}=120+10[\log \mathrm{C}+\log (\ln 2.618)]
$$

With our noise measuring set-up, an index in the range of $+70 \mathrm{~dB}$ down to $-40 \mathrm{~dB}\left(\mathrm{C} \approx 10^{-16}\right)$ can be measured on resistors as low as $10 \Omega$. The $1 / \mathrm{f}$ noise measured on thick-film resistors of the ESL 2900 and 2700 materials is interpreted on the ground of Eqs. (3), (4), (8), (9), (10) and (11). The ESL 2900 is a ruthenium-based resistor system and the nominal sheet resistivity of the resistors ranges from $10^{2} \Omega$ to $10^{5} \Omega$ per square. On average, the resistance deviations from the nominal value was less than $7 \%$. The noise index ranges from $-32.2 \mathrm{~dB}$ to $+10.8 \mathrm{~dB}$ for resistors of $1.25 \times 1.25 \mathrm{~mm}$. For the $10^{4} \Omega$ and $10^{5} \Omega$ resistors the observed ratio between $\mathrm{C}$ for a $10^{5} \Omega$ and $10^{4} \Omega$ resistors is 300 . This could mean that these thick-film resistors are constriction dominated. From Eq. (9) follows that, (assuming that two constriction dominated thick-film resistors have grains with the same $\mathrm{n}$ and $\rho$ values and the same $\gamma$ value.) $C_{1} / C_{2}=\left(R_{\square 1} / R_{\square 2}\right)^{3}$.

By adding more glass to a resistor paste a higher resistance value may be expected owing to the smaller contact radius $a$ of the touching grains (Eq. (8)). For the $10^{2} \Omega$ and $10^{3} \Omega$ resistors the measured ratio between the $C$ values is about 4 . This small value suggests that the $10^{2} \Omega$ and $10^{3} \Omega$ resistors are interface dominated. In this case $t, \mathrm{a}, \gamma$ and even $\mu_{\mathrm{i}}$, which often depends on $t^{12}$, are interrelated. Using Eqs. (10) and (11) and the interrelationship between $t, \alpha, \gamma$ and $\mu_{\mathrm{i}}$ leads to a proportionality $C_{1} / C_{2}=\left(R_{\square 1} / R_{\square 2}\right)^{\beta}$ with $\beta<1$. Out of each of the 2715, 2716 and 2717 ESL series one fired plate was investigated. The observed noise index ranges from $+4.3 \mathrm{~dB}$ to $+14.3 \mathrm{~dB}$ calculated for $1.25 \mathrm{~mm} \times 1.25 \mathrm{~mm}$ size. The results from the 2700 system showed $C_{1} / C_{2}=\left(R_{\square 1} / R_{\square 2}\right)^{\beta}$ with $\beta$ between 1 and 0.8 . This means that the resistor is interface dominated.

The 2700 resistors were on test patterns with resistivity areas in the range of $0.4 \mathrm{~mm}^{2}$ to $20 \mathrm{~mm}^{2}$. Apart from some scattering in noise data, $\mathrm{C}$ was inversely proportional to the areas. The smallest areas often showed too much noise.

The measured noise of AgPd conductors was lower than $-46 \mathrm{~dB}$.

The observed decrease in $R_{\square}$ and increase in $C$ after a high voltage impulse treatment is in agreement with results presented by Stevens, ${ }^{7}$ and is understood in terms of creation of noisy poor conducting channels in parallel with the existing channels before the impulse fritting procedure. The high voltage breakdown of a thick interface layer that insulates two linking branches of contacting grains, can result in a poor conducting and noisy constriction dominated channel. The influence of one or more very small noisy constrictions in parallel with a large number of less noisy channels has been dealt with by Vandamme. ${ }^{15}$

\subsection{Experimental Results on Carbon Sheet Resistors}

From the resistor presented in Figure 1 we may expect some anisotropy in $R_{\square}$ and in $\left\langle\left(\Delta R_{\square} / R_{\square}\right)^{2}\right\rangle$. For an electrode configuration as presented in Figure 1, Eqs. (1) and (2) are rewritten as

$$
\begin{aligned}
& \mathrm{R}=\left(\gamma_{l} / \gamma_{b} \gamma_{d}\right)(l / b d) r \\
& \left.<(\Delta \mathrm{R} / \mathrm{R})^{2}\right\rangle=\left\langle(\Delta \mathrm{r} / \mathrm{r})^{2}\right\rangle\left(\gamma_{l} \gamma_{b} \gamma_{d} l b d\right)
\end{aligned}
$$

Let us consider two electrode configurations, the first as presented in Figure 1 and the second configuration with the electrodes perpendicular to the breadth direction. Assuming $l=b$ and the same thickness $d$ in both situations and using Eqs. (15) and (16), the ratios between the sheet resistors and between the relative noise are written as

$$
\frac{\mathrm{R}_{\square}}{\mathrm{R}_{\square \perp}}=\left(\frac{\gamma_{l}}{\gamma_{b}}\right)^{2} \frac{r}{r_{\perp}}
$$

with $R_{\square \perp}$ the sheet resistance in the breadth direction 
and $r_{\perp}$ the grain contact resistance represented in the dotted area of Figure 1 labelled $\perp$.

$$
\frac{\left.<\left(\Delta \mathrm{R}_{\square} / \mathrm{R}_{\square}\right)^{2}\right\rangle}{\left\langle\left(\Delta \mathrm{R}_{\square \perp} / \mathrm{R}_{\square}\right)^{2}\right\rangle}=\frac{\left.<(\Delta r / r)^{2}\right\rangle}{\left\langle\left(\Delta \mathrm{r}_{\perp} / \mathrm{r}_{\perp}\right)^{2}\right\rangle}=\left(\frac{\mathrm{r}}{\mathrm{r}_{\perp}}\right)^{3}
$$

For contacts between grains dominated by the constriction resistance, ${ }^{10}<(\Delta \mathrm{r} / \mathrm{r})^{2}>$ is proportional to $r^{3}$. In this case the ratio in Eq. $(16)$ equals $\left(r / r_{\perp}\right)^{3}$. For rolled carbon sheet resistors having the rolldirection coinciding with the length direction as presented in Figure 1, we may expect that, owing to the shear force of the rollers, $\gamma_{l}<\gamma_{b}$ and $r<r_{\perp}$. From investigations on carbon sheet resistors for both anisotropy ratios Eqs. (17) and (18) we obtained about 0.76 . Using Eq. (18) leads to a ratio $r / r_{\perp}$ equal to 0.91 and using Eq. (17) with the ratio $r / r_{\perp}=0.91$ we find $\gamma_{l} / \gamma_{b}$ equal to 0.92 . Under the assumptions made, Eqs. (18) and (17) make it possible to calculate $\gamma_{l} / \gamma_{b}$ and $r / r_{\perp}$ from the experimentally obtained anisotropy ratios in the sheet resistance and in the relative noise.

\section{DISCUSSION AND CONCLUSION}

Noise in thick-film resistors is often erroneously interpreted ${ }^{16,21}$ by ignoring the mesh network of contacts between grains. The simple application of Hooge's empirical relation ${ }^{17}$ to granular structures leads to erroneous $\alpha$ values. These calculations are, however, no arguments against the empirical relation. Ringo et.al. ${ }^{16}$ collected published noise data from a number of thick-film resistor material systems and plotted these data versus sheet resistivity $R_{\square}$. The survey made of the data suggests that for those samples the noise is proportional to $R_{\square}$ only for a limited sheet resistance range in Dupont 7800 materials. For his other material systems noise is proportional to $R_{\square}{ }^{\beta}$ with $1 / 3<\beta \leqslant 3$. These investigators used the simple application of the empirical relation, which is not permissible owing to the nonuniform fields in the contacts (constriction dominated) and to the geometric factors $1 / \gamma^{3} l^{2} d$ (Eq. 4) and $1 / \gamma^{2} t^{2}$ (Eq. 11). If, by neglecting the small linking branches, one were to describe the measured noise as a noise which stems from a homogeneous sample then one would find an apparent $\alpha_{\mathrm{p}}$ value from

$$
<\left(\Delta \mathrm{R}_{\square} / \mathrm{R}_{\square}\right)^{2}>=\alpha_{\mathrm{p}} q \mu \mathrm{R}_{\square} \Delta \mathrm{f} / l^{2} \mathrm{f}
$$

Using Eq. (11) for an interface dominated thickfilm resistor one obtains

$$
\alpha_{\mathrm{p}}=\alpha / \gamma^{2} t^{2}
$$

Since the gap distance $t$ between the particles is smaller than the particle diameters $\gamma^{2} t^{2}$ is always smaller than 1. An incorrect $\alpha$-value of 0.3 in thickfilm materials was found by Ringo et.al. ${ }^{16}$ due to overlooking the complicated interconnecting network. For a thick-film constriction dominated resistance an equation similar to Eq. (20) is obtained by using Eqs. (8), (9) and (6).

$$
\alpha_{p}=\alpha / 20 \gamma^{2} a^{2}
$$

Again, since the contact radius $a$ is smaller than the particle diameter, $1 / 20 \gamma^{2} a^{2}>1$. Consequently $\alpha_{p}$ is always greater than $\alpha$.

Our simple model of the noise of a thick-film resistor leads to two limiting cases. For thick-film resistors with a conduction dominated by the glassparticle interface the relative noise is roughly proportional to $R_{\square}$. For thick-film resistors where the conduction is mainly dominated by the current constrictions in the contact areas between grains, the relative noise is proportional to $R_{\square}{ }^{3}$. Both trends have been observed experimentally.

\section{ACKNOWLEDGEMENTS}

We would like to thank Dr. R. Buck (Electro Science Labs. Deutschland) for providing thick-film resistor samples and M. Klein Haneveld for performing some experiments.

\section{REFERENCES}

1. D. A. Bianchi and A. Cattaneo, "Evaluation of the noise index in laser trimmed thick film resistors." IERE Conference Proceedings no. 31. Conf. on Hybrid Microelectr. Sept. 1975, p. 205.

2. A. Kusy, "Measurement of noise properties of $\mathrm{Pd} / \mathrm{PdO} /$ Ag films by Quan tech type meter," Rozpr. Electrotechn. 20, 313 (1974) in Polish.

3. C. Y. Kuo, "The effects of trimming methods on current noise in thick-film resistors," Proceedings 1971, 21st Electr. Compon. Conference p. 389.

4. C. Y. Kuo and H. G. Blank, "The effects of resistor geometry on current noise in thick-film resistors," 1968 Hybrid Microelectron. Symposium, Oct. 1968. O'Hare Inn Rosmont, Illinois, p. 153.

5. F. Maloberti, F. Montechi and V. Svelto, "Flicker noise in thick-film resistors," Alta Frequenza, 44, 681 (1975).

6. L. K. J. Vandamme and R. P. Tijburg, "1/f Noise measurements for characterizing multispot low ohmic contacts," J. Appl. Phys. 47, 2056 (1976).

7. E. H. Stevens, D. A. Gilbert and J. A. Ringo, "Highvoltage damage and low frequency noise in thick-film resistors," IEEE Trans. on Parts, Hybrids and Packaging PHP-12, 351 (1976).

8. J. L. Vossen, "Screening of metal film defects by current noise measurements," Appl. Phys. Lett. 23, 287 (1973). 
9. L. H. F. Ortmans and L. K. J. Vandamme, "Characterization of Impulse-fritting procedures of contacts by measuring 1/f noise," Appl. Phys. 9, 147 (1976).

10. F. N. Hooge and A. M. H. Hoppenbrouwers, "Contact noise," Phys. Lett. A29 642 (1969).

11. L. K. J. Vandamme, "1/f Noise of point contacts affected by uniform films," J. Appl. Phys. 45,4563 (1974).

12. L. K. J. Vandamme, "1/f Noise of InSb point contacts at $77 \mathrm{~K}$ and $300 \mathrm{~K}$," 4th Intern. Confer. on Phys. Aspects of Noise in Solid State Devices, Noordwijkerhout, Sept. 1975.

13. L. K. J. Vandamme, "On the calculation of $1 / \mathrm{f}$ noise of contacts," Appl. Phys. 11, 89 (1976).

14. L. K. J. Vandamme and W. M. G. van Bokhoven, "Conductance noise investigations with four arbitrarily shaped and placed electrodes." Appl. Phys. 14, 205 (1977).

15. L. K. J. Vandamme, "On $1 / \mathrm{f}$ noise in ohmic contacts,"
Doctor thesis, Eindhoven University of Technology, 1976, p. 85-93.

16. J. A. Ringo, E. H. Stevens and D. A. Gilbert, "On the interpretation of Noise in thick film resistors," IEEE Trans. PHP-12, 378 (1976).

17. F. N. Hooge, "1/f Noise," Physica. 83B, 14 (1976).

18. R. Morrison, "Protecting signal circuits by grounding and shielding," Instrumentation Technology, October (1973) 33-36.

19. K. J. Euler, "Das Rauschen von elektrochemischen Stromquellen," ETZ-B, 24, (1972) 115-117.

20. G. T. Conrad, N. Newman and A. P. Stansbury, "A recommended Standard Resistor-Noise Test System," IRE Trans. on Component parts, CP-7, (3) Sept. 1960.

21. A. C. Anderson, J. H. Anderson and M. P. Zaitlin, "Some observations on resistance thermometry below 1 K," Rev. Sci. Instrum., 47, 407 (1976). 

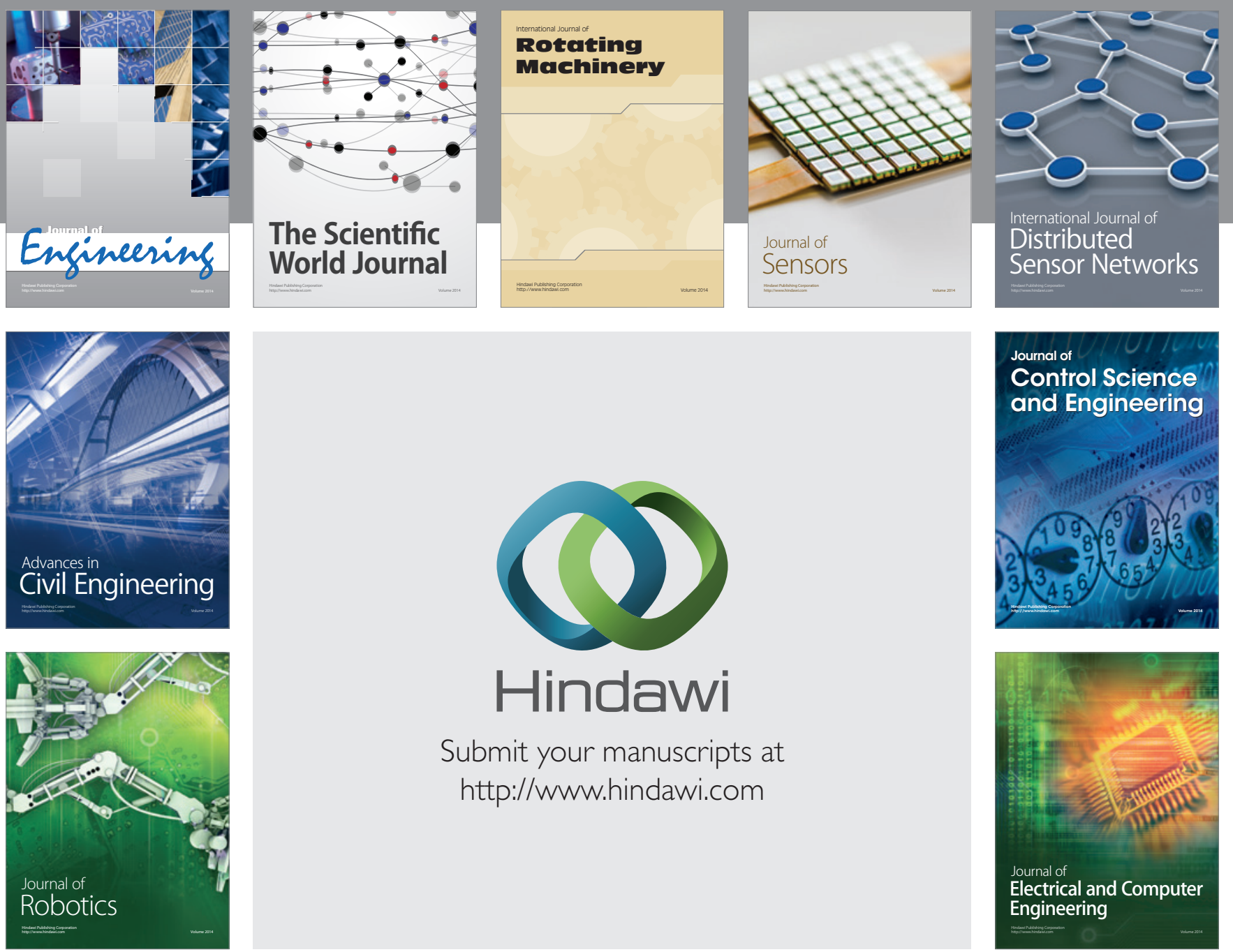

Submit your manuscripts at

http://www.hindawi.com
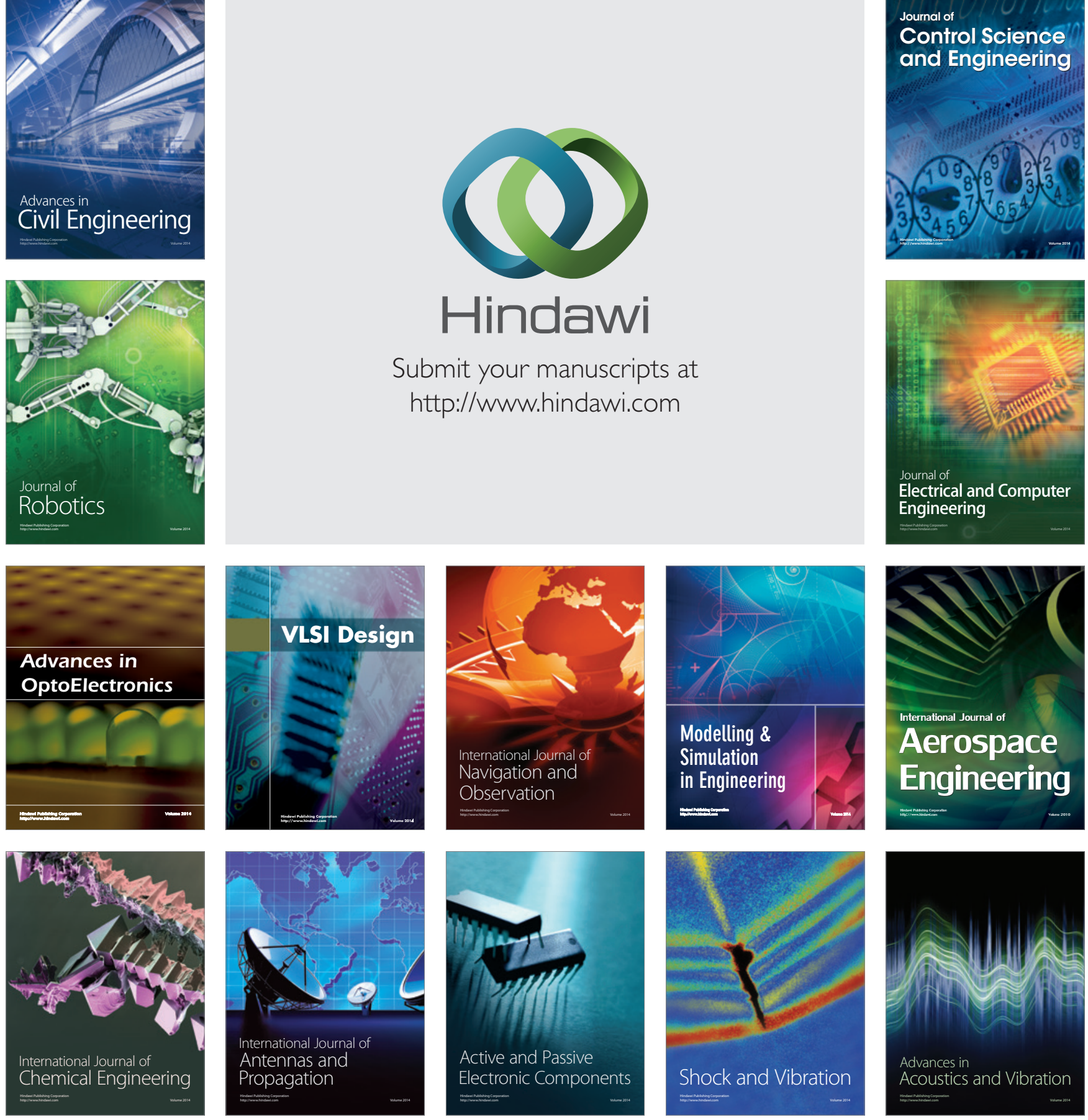